
\title{
is Research Soure \\ Beyond the spine-the spread of ERK and PKA signaling during structural plasticity
}

Shen Tang

Ryohei Yasuda

\section{Video Abstract}

Keywords: ERK, Extracellular signal-regulated kinase, PKA, Protein kinase A, LTP, Long-term potentiation, Spine, Dendrite, Synaptic plasticity, Fluorescence resonance energy transfer, FRET, Two-photon fluorescence lifetime imaging microscropy, 2pFLIM, Memory, Learning, Spatiotemporal dynamics, EKAR, Super rosonance energy-accepting chromoprotein, sREACh, glutamate uncaging

Posted Date: September 20th, 2019

DOI: https://doi.org/10.21203/rs.2.15097/v1

License: (c) (i) This work is licensed under a Creative Commons Attribution 4.0 International License.

Read Full License 


\section{Abstract}

Learning something new not only changes our perspectives and behavior - it actually changes the structure of our brains. Memories and experiences are recorded in the brain by altering the physical connections between neurons. Until recently, however, the protein signals that cause these tiny structural changes were too small to measure with available imaging methods. But researchers at the Max Planck Florida Institute for Neuroscience created ultra-sensitive sensors and revealed the activity of two of the proteins that write memories into neural circuits in the brain. Individual neurons have many branches, or dendrites. And each dendrite can be covered with thousands of tiny bumps called spines, where messages are received from other neurons. Changes in spine size are one way memories are recordedwhen lots of messages are being passed and a spine is very active, it gets bigger. Many proteins need to be activated to make spines grow. Extracellular signal-regulated kinase, or ERK, and protein kinase A, or PKA, are two of the most important. But because the signals put out by existing ERK and PKA sensors were too weak to pick up in spines, scientists weren't sure how ERK and PKA activation is translated into memory-related changes in spine structure. To overcome this limitation, the researchers modified existing sensors so that they output three times more signal, making it possible to measure ERK and PKA activation within single spines. Then they expressed the sensors in hippocampal neurons in mouse brain slices and artificially stimulated individual spines. As expected, stimulation made the spines grow and activated both ERK and PKA. But the ultra sensitive sensors revealed that the kinases weren't active just in the spine - the activity also spread into the dendrite and nearby spines. This indicates that the influence of stimulation spreads to multiple nearby spines and could facilitate growth of those as well. The scientists wanted to be sure the apparent spread wasn't caused by movement of the sensors rather than the spread of kinase activity. So they tethered the sensors to an anchor protein in the spine. ERK and PKA activity spread over a similar distance when they used the anchored sensors, confirming they were witnessing the spread of kinase activity. This activity spillover might serve a useful messaging function in memory formation. Spreading of the signal into the dendrite is likely to trigger the spine-growth-related events that occur there, such as exocytosis of membrane receptors and protein synthesis. In addition, converting temporary changes in spine size into permanent memories requires gene transcription and subsequent production of new proteins, so the spread of kinase activity out of individual spines may help spines and dendrites tell the nucleus to start transcribing genes and build proteins. The unprecedented sensitivity of the new sensors could enable scientists to further define the roles of PKA and ERK in various forms of cellular signaling, such as cell division, differentiation and proliferation as well as synaptic plasticity. And because abnormal PKA and ERK activity can also cause psychiatric disorders and cancer, increased understanding of their signaling may also help improve treatments for countless patients. 